\title{
Dynamical System Analysis of Modified Chaplygin Gas in Einstein-Aether Gravity
}

\author{
Chayan Ranjit1] \\ Department of Mathematics, Seacom Engineering College, Howrah-711 302, India.
}

\begin{abstract}
Prabir Rudra 2
Department of Mathematics, Indian Institute of Engineering Science and Technology, Shibpur, Howrah-711 103 , India. Department of Mathematics, Pailan College of Management and Technology, Bengal Pailan Park, Kolkata-700 104, India.
\end{abstract}

\author{
Sujata Kundu \\ Department of Information Technology, Narula Institute of Technology, Kolkata-700109, India
}

\begin{abstract}
In this work we investigate the background dynamics when dark energy is coupled to dark matter with a suitable interaction in the universe described by Einstein-Aether gravity. Dark energy in the form of Modified Chaplygin gas is considered. A suitable interaction between dark energy and dark matter is considered in order to at least alleviate (if not solve) the cosmic coincidence problem. The dynamical system of equations is solved numerically and a stable scaling solution is obtained. A significant attempt towards the solution of the cosmic coincidence problem is taken. The statefinder parameters are also calculated to classify the dark energy models. Graphs and phase diagrams are drawn to study the variations of these parameters. It is also seen that the background dynamics of modified Chaplygin gas in Einstein-Aether gravity is completely consistent with the notion of an accelerated expansion in the late universe. Finally, it has been shown that the universe follows the power law form of expansion around the critical point.
\end{abstract}

Pacs no : 04.50.Kd, 95.36.+x, 98.80.Cq, 98.80.-k

\section{Introduction}

At the turn of the last century observations from Ia Supernova and Cosmic Microwave Background (CMB)radiation confirmed that our universe is suffering from an accelerated expansion [1, 2, 3, 4, 5, but the physical origin of this acceleration is yet to be known. The standard explanation invokes an unknown "dark energy" component which has the property that positive energy density and negative pressure. Observations indicate that dark energy occupies about $70 \%$ of the total energy of the universe, and the contribution of dark matter is $\sim 26 \%$. This accelerated expansion of the universe has also been strongly confirmed by some other independent experiments like Sloan Digital Sky Survey (SDSS) [6], Baryonic Acoustic Oscillation (BAO) 7], WMAP data analysis [8, 9] etc. Over the past decade there have been many theoretical models for mimicking the dark energy behaviors, such as the simplest (just) cosmological constant in which the equation of state is independent of the cosmic time and which can fit the observations well. This model is the so-called $\Lambda \mathrm{CDM}$, containing a mixture of cosmological constant $\Lambda$ and cold dark matter (CDM). However, two problems arise from this scenario, namely "fine-tuning" and the "cosmic coincidence" problems. In order to solve these two problems, many dynamical dark energy models were suggested, whose equation of state evolves with cosmic time. The scalar field or quintessence [10, 11] is one of the most favored candidate of dark energy which produce sufficient negative pressure to drive acceleration. In order to alleviate the cosmological-constant problems and explain the acceleration expansion, many dynamical dark energy models have been proposed, such as K-essence, Tachyon, Phantom, quintom, Chaplygin gas model, etc [12, 13, 14, 15, 16. Also the interacting dark energy models including Modified Chaplygin gas [17, holographic dark energy model [28, and braneworld model 64 have been proposed. The equation of state of Modified Chaplygin gas is given by,

\footnotetext{
${ }^{1}$ chayanranjit@gmail.com

2 prudra.math@gmail.com

3 sujatakundu10@gmail.com
} 


$$
p=A \rho-\frac{B}{\rho^{\alpha}},
$$

where $p$ and $\rho$ are respectively the pressure and energy density and $0 \leq \alpha \leq 1, A$ and $B$ are positive constants. In Einstein's gravity, the modified Chaplygin gas [17] best fits with the 3 year WMAP and the SDSS data with the choice of parameters $A=0.085$ and $\alpha=1.72435$ which are improved constraints than the previous ones $-0.35<A<0.025$ 36 .

Another possibility is that general relativity is only accurate on small scales and has to be modified on cosmological distances. One of these is modified gravity theories. In this case cosmic acceleration would arise not from dark energy as a substance but from the geometry of space-time i.e. from the dynamics of modified gravity. Modified gravity constitutes an interesting dynamical alternative to $\Lambda \mathrm{CDM}$ cosmology in that it is also able to describe the current cosmic acceleration. The simplest modified gravity is DGP brane-world model [37]. The other alternative approach dealing with the acceleration problem of the Universe is changing the gravity law through the modification of action of gravity by means of using $f(R)$ gravity 38, 39 instead of the Einstein-Hilbert action. Some of these models, such as $1 / R$ and logarithmic models, provide an acceleration for the Universe at the present time [40. Other modified gravity includes $f(T)$ gravity, $f(G)$ gravity, Gauss-Bonnet gravity, Horava-Lifshitz gravity, Brans-Dicke gravity, etc [41, 42, 43, 44, 45. In recent times there have been a lot of research on the background dynamics of different DE models in modified gravity theories in the quest of a standard model of cosmology [46, 47, 48, 49].

In the present work, we concentrate on the generalized Einstein-Aether (EA) theories as proposed by Zlosnik et al [50. 51, which is a generalization of the Einstein-Aether theory developed by Jacobson et al [52, 53. In recent years a lot of work has been done in generalized Einstein-Aether theories [54, 55, 56, 57, 58, 59, 60, 61. In the generalized Einstein-Aether theories by taking a special form of the Lagrangian density of Aether field, the possibility of EinsteinAether theory as an alternative to dark energy model is discussed in detail, that is, taking a special Aether field as a dark energy candidate and it has been found the constraints from observational data 62, 63. Since modified gravity theory may be treated as alternative to dark energy, so Meng et al 62, 63] have not taken by hand any types of dark energy in Einstein-Aether gravity and shown that the gravity may be generates dark energy. Here if we exempt this assumption, so we need to consider the dark energy from outside. So we assume the FRW universe in Einstein-Aether gravity model filled with the dark matter and the modified Chaplygin gas (MCG) type dark energy.

This paper is organized as follows: Section 2 comprises of the general concepts of Einstein-Aether gravity. In Section 3 , we do an extensive study of the dynamical system. In section 4, a detailed graphical analysis for the phase plane is presented. Finally the paper ends with some concluding remarks in section 5 .

\section{Einstein-Aether Gravity Theory}

In order to include Lorentz symmetry violating terms in gravitation theories, apart from some noncommutative gravity models, one may consider existence of preferred frames. This can be achieved admitting a unit timelike vector field in addition to the metric tensor of spacetime. Such a timelike vector implies a preferred direction at each point of spacetime. Here the unit timelike vector field is called the Aether and the theory coupling the metric and unit timelike vector is called the Einstein-Aether theory 52. So Einstein-Aether theory is the extension of general relativity (GR) that incorporates a dynamical unit timelike vector field (i.e., Aether). In the last decade there is an increasing interest in the Aether theory.

The action of the Einstein-Aether gravity theory with the normal Einstein-Hilbert part action can be written in the form [50, 62 .

$$
S=\int d^{4} x \sqrt{-g}\left[\frac{R}{16 \pi G}+\mathcal{L}_{E A}+\mathcal{L}_{m}\right]
$$

where $\mathcal{L}_{E A}$ is the vector field Lagrangian density while $\mathcal{L}_{m}$ denotes the Lagrangian density for all other matter fields. The Lagrangian density for the vector part consists of terms quadratic in the field [50, 62]:

$$
\begin{gathered}
\mathcal{L}_{E A}=\frac{M^{2}}{16 \pi G} F(K)+\frac{1}{16 \pi G} \lambda\left(A^{a} A_{a}+1\right), \\
K=M^{-2} K^{a b}{ }_{c d} \nabla_{a} A^{c} \nabla_{b} A^{d} \\
K^{a b}{ }_{c d}=c_{1} g^{a b} g_{c d}+c_{2} \delta_{c}^{a} \delta_{d}^{b}+c_{3} \delta_{d}^{a} \delta_{c}^{b}
\end{gathered}
$$

where $c_{i}$ are dimensionless constants, $M$ is the coupling constant which has the dimension of mass, $\lambda$ is a Lagrange multiplier that enforces the unit constraint for the time-like vector field, $A^{a}$ is a contravariant vector, $g_{a b}$ is metric tensor and $F(K)$ ia an arbitrary function of $K$. From (1), we get the field equations

$$
\begin{gathered}
G_{a b}=T_{a b}^{E A}+8 \pi G T_{a b}^{m}, \\
\nabla_{a}\left(F^{\prime} J_{b}^{a}\right)=2 \lambda A_{b}
\end{gathered}
$$


where

$$
F^{\prime}=\frac{d F}{d K} \text { and } J_{b}^{a}=2 K_{b c}^{a d} \nabla_{d} A^{c}
$$

Here $T_{a b}^{m}$ is the energy momentum tensor for matter field and $T_{a b}^{E A}$ is the energy momentum tensor for the vector field and they are respectively given as follows: 62 .

$$
T_{a b}^{m}=(\rho+p) u_{a} u_{b}+p g_{a b}
$$

where $\rho$ and $p$ are respectively the energy density and pressure of matter and $u_{a}=(1,0,0,0)$ is the fluid 4 -velocity vector and

with

$$
T_{a b}^{E A}=\frac{1}{2} \nabla_{d}\left[\left(J_{(a}{ }^{d} A_{b)}-J_{(a}^{d} A_{b)}-J_{(a b)} A^{d}\right) F^{\prime}\right]-Y_{(a b)} F^{\prime}+\frac{1}{2} g_{a b} M^{2} F+\lambda A_{a} A_{b}
$$

$$
Y_{a b}=-c_{1}\left[\left(\nabla_{d} A_{a}\right)\left(\nabla^{d} A_{b}\right)-\left(\nabla_{a} A_{d}\right)\left(\nabla_{b} A^{d}\right)\right]
$$

where the subscript $(a b)$ means symmetric with respect to the indices involved and the vector $A^{a}$ is considered to have time-like direction and satisfies $A^{a} A_{a}=-1$ to fix the variation of the action with respect to $\lambda$. The normalized vector field $A^{a}$ has components $(1,0,0,0)$ in the FRW cosmology with homogeneous and isotropic universe filled with perfect fluid.

\section{Dynamical system analysis in Einstein-Aether gravity}

We consider the Friedmann-Robertson-Walker (FRW) metric of the universe as

$$
d s^{2}=-d t^{2}+a^{2}(t)\left[\frac{d r^{2}}{1-k r^{2}}+r^{2}\left(d \theta^{2}+\sin ^{2} \theta d \phi^{2}\right)\right]
$$

where $k(=0, \pm 1)$ is the curvature scalar and $a(t)$ is the scale factor. From equations (3) and (4), we get

$$
K=\frac{3 \beta H^{2}}{M^{2}}
$$

where $\beta=c_{1}+3 c_{2}+c_{3}$ is constant. From eq. (5), we get the modified Friedmann equation for Einstein-Aether gravity as in the following [50, 62]:

$$
\beta\left(-F^{\prime}+\frac{F}{2 K}\right) H^{2}+\left(H^{2}+\frac{k}{a^{2}}\right)=\frac{8 \pi G}{3} \rho
$$

and

$$
\beta \frac{d}{d t}\left(H F^{\prime}\right)+\left(-2 \dot{H}+\frac{2 k}{a^{2}}\right)=8 \pi G(\rho+p)
$$

where $H\left(=\frac{\dot{a}}{a}\right)$ is Hubble parameter. Now we see that if the first expressions of L.H.S. of equations (14) and (15) are zero, we get the usual field equations for Einstein's gravity. So first expressions arise for Einstein-Aether gravity. Also the conservation equation is given by

$$
\dot{\rho}+3 \frac{\dot{a}}{a}(\rho+p)=0
$$

Now, assume that the matter fluid is combination of dark matter and modified Chaplygin gas type dark energy. So $\rho=\rho_{m}+\rho_{c h}$ and $p=p_{m}+p_{c h}$, where $\rho_{m}$ and $p_{m}$ are respectively the energy density and pressure of dark matter and $\rho_{c h}$ and $p_{c h}$ are respectively the energy density and pressure of modified Chaplygin gas. Assume that the dark matter follows the barotropic equation of state $p_{m}=w_{m} \rho_{m}$, where $w_{m}$ is a constant. The equation of state of modified Chaplygin gas (MCG) is given by 17 .

$$
p_{c h}=A \rho_{c h}-\frac{B}{\rho_{c h}^{\alpha}}
$$

where $A>0, B>0$ and $0 \leq \alpha \leq 1$.

As in the present problem the interaction between DE and pressureless DM has been taken into account for interacting DE and DM the energy balance equation will be

$$
\begin{gathered}
\dot{\rho}_{c h}+3 H\left(1+\omega_{c h}\right) \rho_{c h}=-Q \\
\dot{\rho}_{m}+3 H \rho_{m}=Q
\end{gathered}
$$

where $Q=3 b H \rho$ is the interaction term, $b$ is the coupling parameter (or transfer strength) and $\rho=\rho_{c h}+\rho_{m}$ is the total cosmic energy density which satisfies the energy conservation equation $\dot{\rho}+3 H(\rho+p)=0$ [18, 19].

Since we lack information about the fact, how does DE and DM interact so we are not able to estimate the interaction term from the first principles. However, the negativity of $Q$ immediately implies the possibility of having negative DE in the early universe which is overruled by the necessity of the second law of thermodynamics to be held [20]. Hence $Q$ must 
be positive and small. From the observational data of 182 Gold type Ia supernova samples, CMB data from the three year WMAP survey and the baryonic acoustic oscillations from the Sloan Digital Sky Survey, it is estimated that the coupling parameter between DM and DE must be a small positive value (of the order of unity), which satisfies the requirement for solving the cosmic coincidence problem and the second law of thermodynamics 21. Due to the underlying interaction, the beginning of the accelerated expansion is shifted to higher redshifts. The continuity equations for dark energy and dark matter are given in equations (18) and (19). Now we proceed to study the dynamical system.

\subsection{Dynamical System Analysis}

In this subsection we plan to analyze the dynamical system. For that firstly we convert the physical parameters into some dimensionless form, given by

$$
x=\ln a, \quad u=\frac{\rho_{c h}}{3 H^{2}}, \quad v=\frac{\rho_{m}}{3 H^{2}}, \quad y=\frac{a}{3 H^{2}}
$$

where the present value of the scale factor $a_{0}=1$ is assumed.

Following the works of Zlosnik et al and Zuntz et al [22, 23, we assume the ansatz for $F$ as

$$
F(K)=f_{0} K^{n}, \quad K>0
$$

where cosmic acceleration is realized for $f_{0}>0$ and for certain value of $\mathbf{n}(n \leq 1)$ without the need of a source term in the modified Einstein equations [23, here modelled with the Chaplygin gas. This form is efficient enough to express a wide range of behaviour. Following modified newtonian dynamics, if we use the same branch of $F$ as it uses on small scales, it can be shown that the background cosmology becomes inconsistent with the late time cosmic acceleration [23]. Hence we use a reasonable form for $F$ given in eqn. (27), which works for the accelerated regime $(|K| \gg 1)$. Using this form of $F$, the modified Friedmann equations become,

$$
\left[1+\epsilon\left(\frac{H}{M}\right)^{2(n-1)}\right] H^{2}=\frac{8 \pi G}{3} \rho
$$

where $\epsilon=(1-2 n) f_{0}(-3 \beta)^{n} / 6$. Moreover the expression for $f_{0}$ is obtained as,

$$
f_{0}=\frac{6\left(\Omega_{m}-1\right)}{(1-2 n)(-3 \beta)^{n}}\left(\frac{M}{H_{0}}\right)^{2(n-1)}
$$

where $\Omega_{m}=8 \pi G \rho_{0} / 3 H_{0}^{2}$ and $H_{0}$ is the present value of Hubble constant.

Let us consider some special cases of $n$. We see that if $n=1 / 2$, the Friedmann equations are unchanged $(\epsilon=0)$, and invariably there is no effect on the background cosmology. If $n=0$, the cosmological constant is recovered. When $n=1$, we have $\epsilon=f_{0} \beta / 2$ and there is a change of scale for the Newton's constant by a factor of $1 /(1+\epsilon)$ [24]. A schematic representation of the late time evolution of the universe depending on the value of $n$ can be found in [25]. From the above discussion the most suitable choice of $n$ for our present assignment is $n=1$. Moreover it provides a bit of mathematical simplicity as well. This value of $n$ completely lies in the accepted region which is $n \leq 1$. As apparently conceived, this chosen value of $n$ does not actually hamper the generality of the problem. In fact from our analysis we have seen that a slight variation in the value of $n$ does not bring about significant alterations to the final result.

Using eqns. (13-19) into eqn.(20) we get the parameter gradients as

and

$$
\begin{aligned}
& \frac{d u}{d x}=\frac{H \rho_{c h}^{\cdot}-2 \rho_{c h} \dot{H}}{3 H^{4}} \\
& \frac{d v}{d x}=\frac{H \rho_{m}-2 \rho_{m} \dot{H}}{3 H^{4}}
\end{aligned}
$$

$$
\frac{d y}{d x}=\frac{H \dot{a}-2 a \dot{H}}{3 H^{4}}
$$

where $\omega_{c h}$ is the EoS parameter for MCG determined as

$$
\begin{gathered}
\omega_{c h}=\frac{p_{c h}}{\rho_{c h}}=A-\frac{B}{\left(3 H^{2} u\right)^{\alpha+1}} \\
\dot{H}=\frac{M^{2}}{18 f_{0} \beta^{2} H^{2}-2 M^{2}}\left[8 \pi G\left(3 H^{2}(u(A+1)+v)-\frac{B}{\left(3 H^{2} u\right)^{\alpha}}\right)-\frac{2 k}{a^{2}}\right]
\end{gathered}
$$

Here $H$ is given by,

$$
H=\sqrt{\frac{M^{2}}{9 f_{0} \beta}-\frac{8 G M^{2} \pi u}{9 f_{0} \beta}-\frac{8 G M^{2} \pi v}{9 f_{0} \beta}+\frac{\sqrt{\left(-2 a^{2} M^{2}+16 a^{2} G M^{2} \pi u+16 a^{2} G M^{2} \pi v\right)^{2}+72 a^{2} f_{0} k M^{2} \beta}}{18 a^{2} f_{0} \beta}}
$$




\subsection{Critical Points}

The critical points of the above system are obtained by putting $\frac{d u}{d x}=\frac{d v}{d x}=\frac{d y}{d x}=0$. But due to the complexity of these equations, it is not possible to find a solution in terms of all the involved parameters. So we are compelled to search for a viable numerical solution. In order to accomplish this, we have to replace the parameters by suitable numerical values which must be realistic cosmologically. As far as the parameters of MCG are concerned, we will be using the values which are consistent with the observational data [17, 26, 27]. We consider $A=1 / 3, \quad B=0.5, \quad \alpha=1$. The choice of the Aether gravity parameters will not be so straightforward, since we do not have cosmologically constrained parametric values at our disposal. In [25] Clifton et al has shown that in order to realize a more exotic but viable scenario, one should have $c_{1}=c_{3}=0$. From eqn.(23), we get an idea of the value of $\beta$. If we take $\Omega_{m}=0.3$ (acceptable value from the latest observational data) and $n=1$, we see that in order to make $f_{0}>0, \beta$ should be restricted to negative values. Although we get an idea about the sign of $\beta$ yet there is no information available in literature about the magnitude. So keeping in mind the simplicity of calculations, we choose $\beta=-1$. Using the accepted values of parameters in eqn.(23) we obtain $f_{0}=1.5$ approximately. Since $M$ has the dimensions of mass, so it is obvious that it should have large values. The exact values of $M$ and $b$ are obtained from numerical fine tuning after putting the accepted numerical values of other parameters. We take, $M=5000, \quad b=1$ and obtain the following critical point,

$$
u_{c}=4.32 \times 10^{-14}, \quad v_{c}=1.5, \quad y_{c}=3.89729 \times 10^{-18}
$$

The critical point correspond to the era dominated by DM and MCG type DE. For the critical point $\left(u_{c}, v_{c}\right)$, the equation of state parameter given by equation (24) of the interacting DE takes the form

$\omega_{c h}=A-B\left[3 u_{c}\left\{\frac{M^{2}}{9 f_{0} \beta}-\frac{8 G M^{2} \pi u_{c}}{9 f_{0} \beta}-\frac{8 G M^{2} \pi v_{c}}{9 f_{0} \beta}+\frac{\sqrt{\left(-2 a^{2} M^{2}+16 a^{2} G M^{2} \pi u_{c}+16 a^{2} G M^{2} \pi v_{c}\right)^{2}+72 a^{2} f_{0} k M^{2} \beta}}{18 a^{2} f_{0} \beta}\right\}\right.$

\subsection{Stability Around Critical Point}

Now we check the stability of the dynamical system (eqs. (21) and (22) and (23)) about the critical point. In order to do this, we linearize the governing equations about the critical point i.e.,

$$
u=u_{c}+\delta u, \quad v=v_{c}+\delta v, \quad y=y_{c}+\delta y
$$

Now if we assume $f=\frac{d u}{d x}, g=\frac{d v}{d x}$ and $h=\frac{d y}{d x}$ then we may obtain

$$
\begin{gathered}
\delta\left(\frac{d u}{d x}\right)=\left[\partial_{u} f\right]_{c} \delta u+\left[\partial_{v} f\right]_{c} \delta v+\left[\partial_{y} f\right]_{c} \delta y \\
\delta\left(\frac{d v}{d x}\right)=\left[\partial_{u} g\right]_{c} \delta u+\left[\partial_{v} g\right]_{c} \delta v+\left[\partial_{y} g\right]_{c} \delta y
\end{gathered}
$$

and

$$
\delta\left(\frac{d y}{d x}\right)=\left[\partial_{u} h\right]_{c} \delta u+\left[\partial_{v} h\right]_{c} \delta v+\left[\partial_{y} h\right]_{c} \delta y
$$

where

$$
\begin{gathered}
\begin{array}{r}
\partial_{u} f=\frac{1}{3 f_{0} u \beta} 2^{1-\alpha} M^{2}\left(-\frac{M^{2} u(-1+u+v)}{f_{0} \beta}\right)^{-\alpha}\left(3^{1+\alpha} B f_{0} \beta\left(-u(-1+2 u+v)(-1+\alpha)+3 f_{0} y(-u+(-1+2 u+v) \alpha) \beta\right)\right. \\
-2^{1+\alpha} f_{0}\left(-\frac{M^{2} u(-1+u+v)}{f_{0} \beta}\right)^{1+\alpha} \beta\left(4(1+A) u^{2}+3(1+A+b) f_{0} y \beta+u\left(-2-2 A+5 v+2 A v-9(1+A+b) f_{0} y \beta\right)\right. \\
\left.\left.+v\left(-1+v-3(1+A+3 b) f_{0} y \beta\right)\right)\right)
\end{array} \\
\begin{array}{r}
\partial_{v} f=-2^{1-\alpha} 3^{\alpha} B M^{2}(-1+\alpha)\left(-\frac{M^{2} u(-1+u+v)}{f_{0} \beta}\right)^{-\alpha}\left(u-3 f_{0} y \beta\right) \\
\end{array}
\end{gathered}
$$




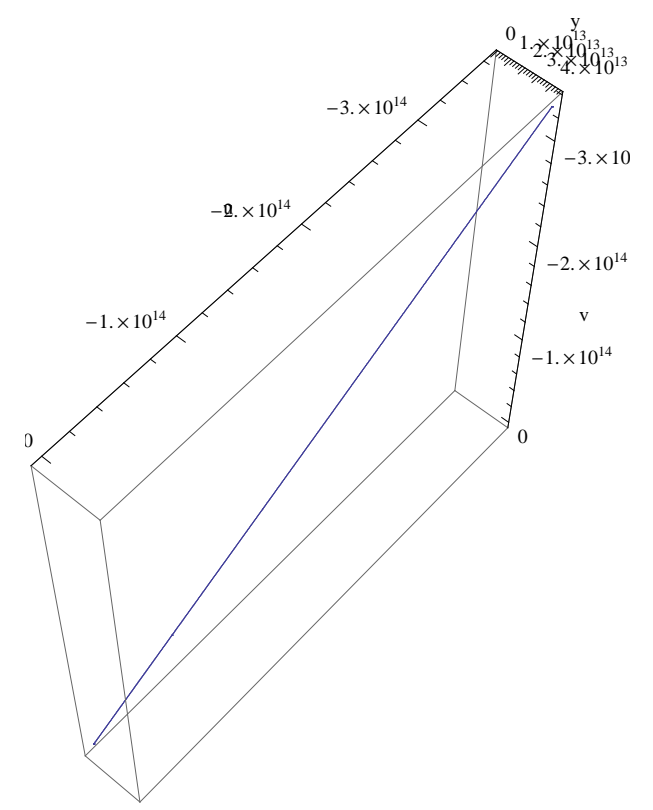

Fig.1

Fig 1: The dimensionless density parameters $u, v$ and $y$ are plotted against each other in a 3D-scenario. Other parameters are fixed at $\alpha=1, \beta=-1, b=1, A=1 / 3, B=0.5, f_{0}=1.2$ and $M=5000$. 


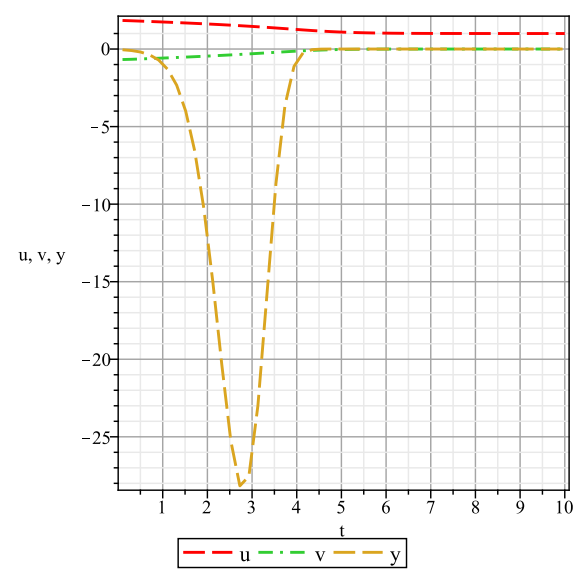

Fig.2

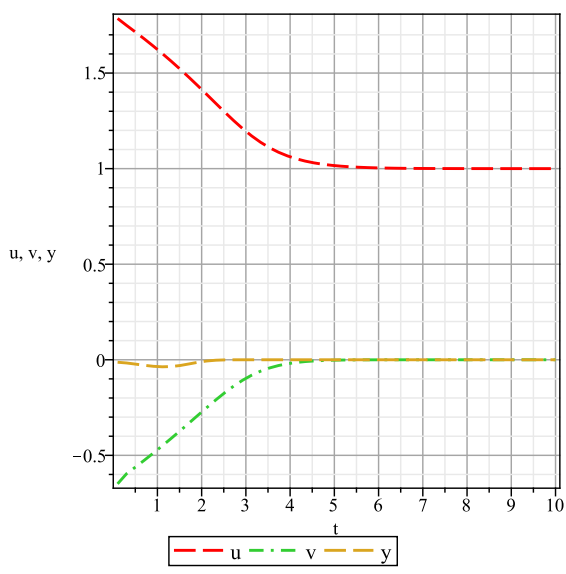

Fig.3

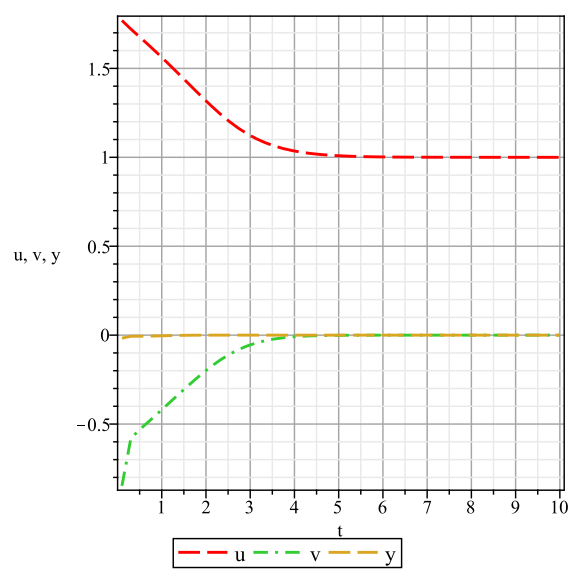

Fig.4

Fig 2 : The dimensionless density parameters are plotted against e-folding time. The initial conditions are $u(0.01)=2.5, v(0.01)=0.02$ and $y(0.01)=0.01$. Other parameters are fixed at $\alpha=1, \beta=-1, b=0.3, A=1 / 3, B=0.5, f_{0}=1.2$ and $M=2000$.

Fig 3 : The dimensionless density parameters are plotted against e-folding time. The initial conditions are $u(0.01)=2.5, v(0.01)=0.02$ and $y(0.01)=0.01$. Other parameters are fixed at $\alpha=1, \beta=-1, b=0.4, A=1 / 3, B=0.5, f_{0}=1.2$ and $M=2000$.

Fig 4 : The dimensionless density parameters are plotted against e-folding time. The initial conditions are $u(0.01)=2.5, v(0.01)=0.02$ and $y(0.01)=0.01$. Other parameters are fixed at $\alpha=1, \beta=-1, b=0.5, A=1 / 3, B=0.5, f_{0}=1.2$ and $M=2000$.

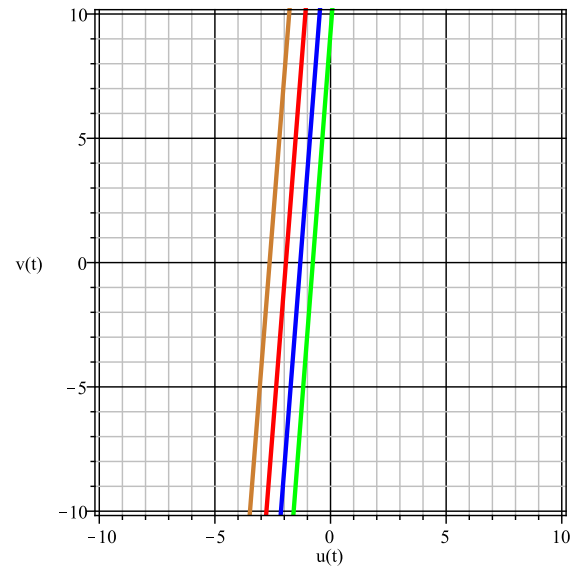

Fig.5

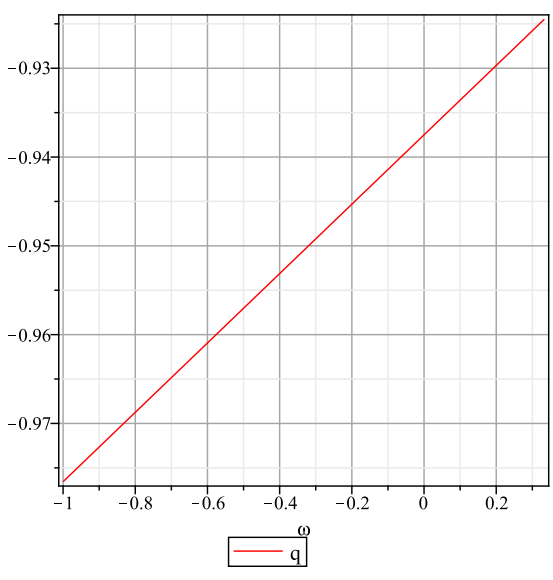

Fig.6

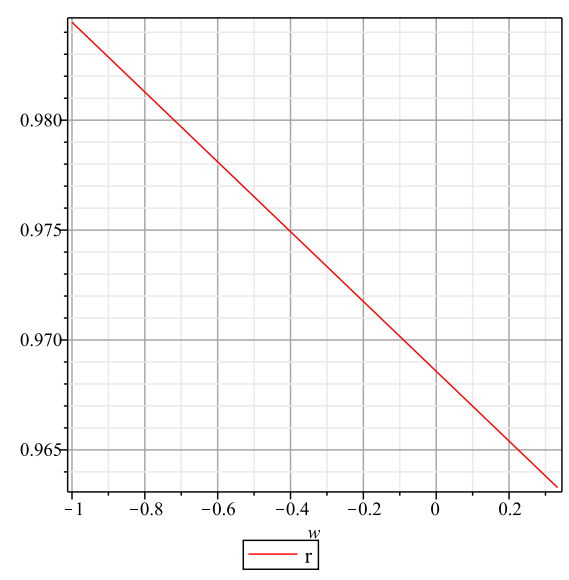

Fig.7

Fig 5: The phase diagram of the parameters $u(t)$ and $v(t)$ depicting an attractor solution. The initial conditions chosen are $u(1)=2.5, v(1)=0.05, y(1)=1.8$ (green); $u(1)=2.6, v(1)=0.06, y(1)=1.9$ (blue); $u(1)=2.7, v(1)=0.07, y(1)=2.0$ (red); $u(1)=2.8, v(1)=0.08, y(1)=2.1$ (gold). Other parameters are fixed at $\alpha=1, \beta=-1, b=1, A=1 / 3, B=0.5, f_{0}=1.2$ and $M=2000$.

Fig 6 : The deceleration parameter is plotted against the EoS parameter. Other parameters are fixed at $\alpha=1, \beta=-1, b=1, A=1 / 3, B=0.5, f_{0}=1.2$ and $M=2000$.

Fig 7 : The statefinder parameter $r$ is plotted against the EoS parameter. Other parameters are fixed at $\alpha=1, \beta=-1, b=1, A=1 / 3, B=0.5, f_{0}=1.2$ and $M=2000$. 


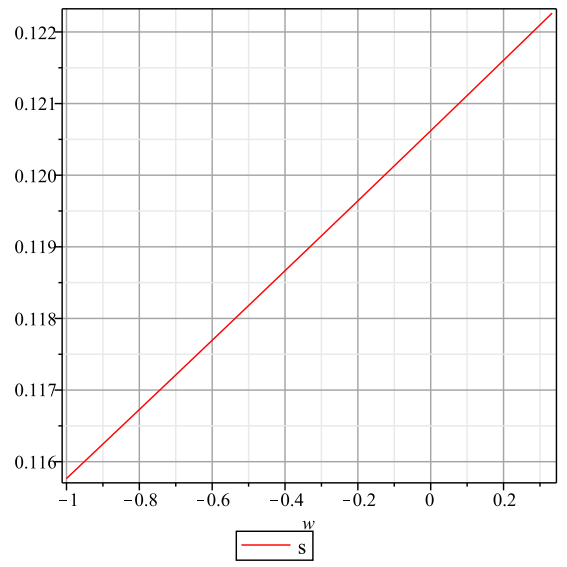

Fig. 8

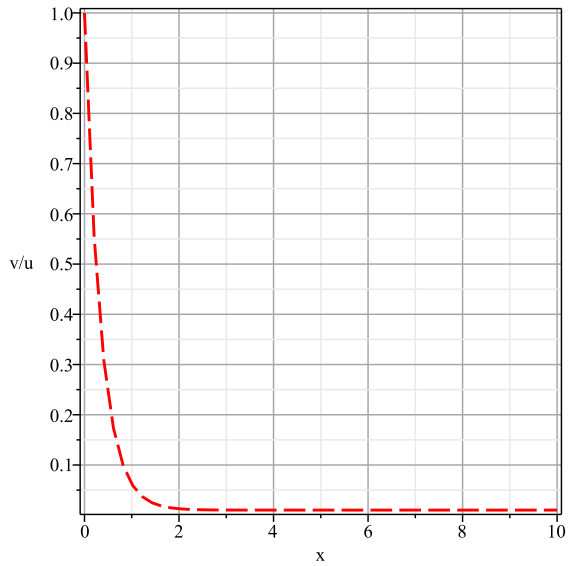

Fig.9

Fig 8: The statefinder parameter $s$ is plotted against the EoS parameter. Other parameters are fixed at $\alpha=1, \beta=-1, b=1, A=1 / 3, B=0.5, f_{0}=1.2$ and $M=2000$.

Fig 9 : The ratio of density parameters is shown against e-folding time. The initial conditions chosen are $v(1)=0.05, u(1)=2.5, y(1)=1.8$. Other parameters are fixed at $\alpha=1, \beta=-1, b=1, A=1 / 3, B=0.5, f_{0}=1.2$ and $M=2000$.

$$
+\frac{4 M^{4}(-1+u+v)\left(u(-1+(3+2 A) u+3 v)-3 f_{0}(2(1+A) u+b(-1+3 u+3 v)) y \beta\right)}{3 f_{0} \beta}
$$

$$
\partial_{y} f=2 M^{2}(-1+u+v)\left(-2 M^{2}(-1+u+v)((1+A+b) u+b v)-2^{-\alpha} 3^{1+\alpha} B f_{0}\left(-\frac{M^{2} u(-1+u+v)}{f_{0} \beta}\right)^{-\alpha} \beta\right)
$$

$$
\begin{aligned}
& \partial_{u} g=-\frac{1}{u(1+2(-1+u+v) \beta)^{2}} 2^{1-\alpha} M^{2} y\left(-\frac{M^{2} u(-1+u+v)}{f_{0} \beta}\right)^{-\alpha} \\
& \left(2^{1+\alpha} b u(-1+u+v)(-1+3 u+3 v)\left(-\frac{M^{2} u(-1+u+v)}{f_{0} \beta}\right)^{\alpha}(M+2 M(-1+u+v) \beta)^{2}+\right. \\
& v\left(3^{1+\alpha} B f_{0} \beta(-u-\alpha+2 u \alpha+v \alpha+2(-1+u+v)(-1+2 u+v) \alpha \beta)+2^{1+\alpha} f_{0}\left(-\frac{M^{2} u(-1+u+v)}{f_{0} \beta}\right)^{1+\alpha} \beta\right. \\
& \left.\left.\left(-(-1+u+v)(-1+2 \beta)(3+4(-1+u+v) \beta)+A\left(-1+v+4 u^{2} \beta+2(-1+v)^{2} \beta+u(3+6(-1+v) \beta)\right)\right)\right)\right)
\end{aligned}
$$

$$
\partial_{v} g=-\frac{1}{(1+2(-1+u+v) \beta)^{2}} 2^{1-\alpha} M^{2} y\left(-\frac{M^{2} u(-1+u+v)}{f_{0} \beta}\right)^{-\alpha}
$$

$$
\begin{gathered}
\left(-3^{1+\alpha} B f_{0} \beta(-1+u+2 v-v \alpha+2(-1+u+v)(-1+u+v-v \alpha) \beta)+\right. \\
2^{1+\alpha} M^{2}(-1+u+v)\left(-\frac{M^{2} u(-1+u+v)}{f_{0} \beta}\right)^{\alpha}\left(-1+2 u+A u-u^{2}-A u^{2}+5 v-5 u v-3 A u v-4 v^{2}-\right. \\
2(-1+u+v)\left((-1+u)(-2+u+A u)+2(-4+(2+A) u) v+3 v^{2}\right) \beta+4(-1+u+v)^{2}(-1+u+3 v) \beta^{2}+ \\
\left.\left.b(-1+3 u+3 v)(1+2(-1+u+v) \beta)^{2}\right)\right)
\end{gathered}
$$




$$
\begin{aligned}
& \partial_{y} g=2 M^{2}(-1+u+v)\left(-2 b M^{2}(-1+u+v)(u+v)+\frac{1}{1+2(-1+u+v) \beta}\right. \\
& \left.\times v\left(2^{-\alpha} 3^{1+\alpha} B f_{0}\left(-\frac{M^{2} u(-1+u+v)}{f_{0} \beta}\right)^{-\alpha} \beta+2 M^{2}(-1+u+v)(-1+u+A u+v-2(-1+u+v) \beta)\right)\right) \\
& \partial_{u} h=\frac{1}{u(1+2(-1+u+v) \beta)^{2}} 2^{1-\alpha} M^{2} y\left(-\frac{M^{2} u(-1+u+v)}{f_{0} \beta}\right)^{-\alpha} \\
& \left(-3^{2+\alpha} B f_{0} \beta(-u-\alpha+2 u \alpha+v \alpha+2(-1+u+v)(-1+2 u+v) \alpha \beta)-2^{1+\alpha} f_{0}\left(-\frac{M^{2} u(-1+u+v)}{f_{0} \beta}\right)^{1+\alpha} \beta\right. \\
& \left.\left(-1-3 A+9 u+9 A u+9 v+3 A v+2(-1+u+v)(1+6 u+6 v+3 A(-1+2 u+v)) \beta+8(-1+u+v)^{2} \beta^{2}\right)\right) \\
& \partial_{v} h=\frac{1}{(1+2(-1+u+v) \beta)^{2}} 2^{1-\alpha} M^{2} y\left(-\frac{M^{2} u(-1+u+v)}{f_{0} \beta}\right)^{-\alpha} \\
& \left(-3^{2+\alpha} B f_{0} \beta(-1+\alpha+2(-1+u+v) \alpha \beta)+2^{1+\alpha} M^{2}(-1+u+v)\left(-\frac{M^{2} u(-1+u+v)}{f_{0} \beta}\right)^{\alpha}\right. \\
& \left.\left(-1+9 u+6 A u+9 v+2(-1+u+v)(1+3(2+A) u+6 v) \beta+8(-1+u+v)^{2} \beta^{2}\right)\right) \\
& \partial_{y} h=-\frac{1}{1+2(-1+u+v) \beta} 2 M^{2}(1-u-v) \\
& \left(2^{-\alpha} 3^{2+\alpha} B f_{0}\left(-\frac{M^{2} u(-1+u+v)}{f_{0} \beta}\right)^{-\alpha} \beta+2 M^{2}(-1+u+v)(1+3 u+3 A u+3 v+2(-1+u+v) \beta)\right)
\end{aligned}
$$

The Jacobian matrix of the above system is given by,

$$
J_{(u, v, y)}^{(E A)}=\left(\begin{array}{lll}
\frac{\delta f}{\delta u} & \frac{\delta f}{\delta v} & \frac{\delta f}{\delta y} \\
\frac{\delta g}{\delta u} & \frac{\delta g}{\delta v} & \frac{\delta g}{\delta y} \\
\frac{\delta h}{\delta u} & \frac{\delta h}{\delta v} & \frac{\delta h}{\delta y}
\end{array}\right)
$$

The eigen values of the above matrix are calculated at the critical point $\left(u_{c}, v_{c}, y_{c}\right)$ and are found to be

$\lambda_{1}=1.40623 \times 10^{15}, \quad \lambda_{2}=1.56236 \times 10^{14}, \quad \lambda_{3}=-0.00543761$.

Hence it is a Saddle Point.

\subsection{Nature of cosmological parameters}

In EA model, the deceleration parameter $q$ can be obtained as

$$
\begin{gathered}
q^{(E A)}=-1-\frac{3}{2} \\
\times \frac{\left\{2 M^{2}\left(-\frac{2 k}{a^{2}}+8 G \pi(p+\rho)\right)\right\}}{\left[3\left\{\frac{M^{2}}{9 f_{0} \beta}+\frac{\sqrt{a^{4} M^{4}+18 a^{2} f_{0} k M^{2} \beta+48 a^{4} f_{0} G M^{2} \pi \beta \rho}}{9 a^{2} f_{0} \beta}\right\}\left\{-2 M^{2}+18 f_{0} \beta^{2}\left(\frac{M^{2}}{9 f_{0} \beta}+\frac{\sqrt{a^{4} M^{4}+18 a^{2} f_{0} k M^{2} \beta+48 a^{4} f_{0} G M^{2} \pi \beta \rho}}{9 a^{2} f_{0} \beta}\right)\right\}\right]}
\end{gathered}
$$

From above we get,

$$
q_{c}^{(E A)}=-1+\frac{3}{2} X_{(E A)}
$$


where

$$
\begin{gathered}
X_{(E A)}= \\
-\frac{\left\{2 M^{2}\left(-\frac{2 k}{a^{2}}+8 G \pi(p+\rho)\right)\right\}}{\left[3\left\{\frac{M^{2}}{9 f_{0} \beta}+\frac{\sqrt{a^{4} M^{4}+18 a^{2} f_{0} k M^{2} \beta+48 a^{4} f_{0} G M^{2} \pi \beta \rho}}{9 a^{2} f_{0} \beta}\right\}\left\{-2 M^{2}+18 f_{0} \beta^{2}\left(\frac{M^{2}}{9 f_{0} \beta}+\frac{\sqrt{a^{4} M^{4}+18 a^{2} f_{0} k M^{2} \beta+48 a^{4} f_{0} G M^{2} \pi \beta \rho}}{9 a^{2} f_{0} \beta}\right)\right\}\right]}
\end{gathered}
$$

If $\quad \frac{2 k}{a^{2}}=8 \pi G(p+\rho), \quad X_{(E A)}=0, \quad$ we have $q=-1$, which confirms the accelerated expansion of the universe. When $M^{2}=9 f_{0} \beta^{2}\left(\frac{M^{2}}{9 f_{0} \beta}+\frac{\sqrt{a^{4} M^{4}+18 a^{2} f_{0} k M^{2} \beta+48 a^{4} f_{0} G M^{2} \pi \beta \rho}}{9 a^{2} f_{0} \beta}\right)$ we have $q=-\infty$. Therefore we have super accelerated expansion of the universe.

In this scenario, the Hubble parameter can be obtained as,

$$
H=\frac{2}{3 X_{(E A)} t}
$$

where the integration constant has been ignored. Integration of eqn.(44) yields

$$
a(t)=a_{0} t^{\frac{2}{3 X_{(E A)}}}
$$

which gives the power law form of expansion of the universe. In order to have an accelerated expansion of universe in EA gravity we must have $0<X_{(E A)}<\frac{2}{3}$. Using this range of $X_{(E A)}$ in the equation $q_{c}^{E A}=-1+\frac{3}{2} X_{(E A)}$, we get the range of $q_{c}^{(E A)}$ as $-1<q_{c}^{(E A)}<0$. This is perfectly consistent with an accelerated expansion of the universe.

Sahni et al (2003) 64 introduced a pair of cosmological diagnostic pair $\{r, s\}$ which is known as as statefinder parameters. The two parameters are dimensionless and are geometrical since they are derived from the cosmic scale factor alone. Also this pair generalizes the well-known geometrical parameters like the Hubble parameter and the deceleration parameter. The statefinder parameters are given by

$$
r \equiv \frac{\dddot{a}}{a H^{3}}, \quad s \equiv \frac{r-1}{3(q-1 / 2)}
$$

In EA model, we have the following expressions of $r$ and $s$ as

$$
r_{(E A)}=\left(1-\frac{3 X_{(E A)}}{2}\right)\left(1-3 X_{(E A)}\right) .
$$

and

$$
s_{(E A)}=X_{(E A)}
$$

\section{Graphical Analysis}

Graphs are obtained and phase diagrams are drawn in order to determine the type of critical point obtained in this model. Below we discuss the results obtained in detail:

The dimensionless density parameters $u$ and $v$ and $y$ are plotted against each other in figure 1 . In figs. 2,3 and 4, the density parameters are plotted against time for different values of interacting coupling parameter. From the figures we see that $u$ always dominates over $v$ during evolution of the universe. So this result is consistent with the well known idea of an energy dominated universe.

Fig. 5 shows the phase portrait of the density parameters of DE and DM. As already stated before that the critical point obtained in this system is a Saddle point and hence there always remains a question on the stability of the system. In fig.6, a plot of deceleration parameter, $q$ is obtained against the EoS parameter, $\omega$. It is seen that $q$ remains in the negative level thus confirming the recent cosmic acceleration. Figs. 7 and 8 show the plot of the statefinder parameters $r$ and $s$ respectively against the EoS parameter, $\omega$. It is known that in case of $\Lambda$ CDM model $r=1$ and $s=0$. comparing figs. 7 and 8 , it can be seen that the values of $r$ is quite different from 1 corresponding to the values of $\omega$ when $s=0$.

This gives the deviation of the model from the $\Lambda$ CDM model. Finally in fig. 9 , the ratio $v / u$ is plotted against $x=\ln a$. The decreasing trajectory confirms the existence of an energy dominated universe with progressive values of scale factor.

\section{Discussions and Concluding Remarks}

In this work, we have considered a combination of Modified Chaplygin gas in Einstein-Aether gravity model. Our basic idea was to study the background dynamics of MCG in detail when it is incorporated in Aether gravity. Dynamical system analysis was carried out, critical points were found and the stability of the system around those critical points 
was tested. Graphical analysis was done to get an explicit picture of the outcome of the work. In order to find a solution for the cosmic coincidence problem, a suitable interaction between DE and DM was considered. The set of equations characterizing the dynamical system was formed and a stable scaling solution was obtained. Hence this work can be considered to be a significant one as far as solution of cosmic coincidence problem is concerned. From the above analysis we conclude that the combination of MCG in EA gravity makes a perfect model for the expanding universe undergoing a late acceleration.

\section{Acknowledgements}

The authors sincerely acknowledge the facilities provided by the Inter-University Centre for Astronomy and Astrophysics (IUCAA), pune, India where a part of the work was carried out. The authors also thank the anonymous referee for his or her constructive comments that helped them to improve the quality of the manuscript.

\section{References}

[1] Perlmutter, S. J. et al :- Nature 39151 (1998).

[2] Riess, A. G. et al. :- [Supernova Search Team Collaboration] Astron. J. 1161009 (1998).

[3] Riess, A. G. et al. :- Astrophys. J. 607665 (2004).

[4] Bennet, C. et al, :- Phys. Rev. Lett. 852236 (2000).

[5] Spergel, D. N. et al. :- Astrophys. J. Suppl. Ser. 170377 (2007).

[6] Adelman-McCarthy, J. K. et al. :- Astrophys. J. Suppl. Ser. 175297 (2008).

[7] Eisenstein, D. J. et al[SDSS Collaboration], 2005, Astrophys. J. 633,560.

[8] Briddle, S. et al :- Science 2991532 (2003).

[9] Spergel, D. N. et al. :- Astrophys. J. Suppl. 148175 (2003).

[10] Peebles, P. J. E., Ratra, B. :- Astrophys. J. 325 L17 (1988).

[11] Caldwell, R. R., Dave, R., Steinhardt, P. J. :- Phys. Rev. Lett. 801582 (1998).

[12] Armendariz - Picon, C., Mukhanov, V. F., Steinhardt, P. J. :- Phys. Rev. Lett. 854438 (2000).

[13] Sen, A. :- JHEP 0207065 (2002).

[14] Caldwell, R. R. :- Phys. Lett. B 54523 (2002).

[15] Feng, B., Wang, X. L., Zhang, X. M. :- Phys. Lett. B 60735 (2005).

[16] Kamenshchik, A. Y., Moschella, U., Pasquier, V. :- Phys. Lett. B 511265 (2001).

[17] Debnath, U., Banerjee, A. and Chakraborty, S. :- Class. Quantum Grav. 215609 (2004).

[18] Guo, Z.-K., Zhang, Y.-Z. :- Phys. Rev. D 71, 023501(2005).

[19] del Campo, S., Herrera, R., Pavon, D. :- JCAP 0901 020(2009).

[20] Alcaniz, J. S., Lima, J. A. S. :- Phys. Rev. D 72 063516(2005).

[21] Feng, C. et. al. :- Phys. Lett. B 665 111(2008).

[22] Zlosnik, T.G., Ferreira, P.G., Starkman, G.D. :- Phys. Rev. D 75044017 (2007)

[23] Zuntz, J., Zlosnik, T. G., Bourliot, F., Ferreira, P. G., Starkman, G. D. :- Phys. Rev. D 81 104015 (2010).

[24] Carroll, S. M., Lim, E. A.: Lorentz violating vector field slow the universe down :- Phys. Rev. D 70(12) 123525 (2004) arXiv:hep-th/0407149

[25] Clifton, T., Ferreira, P. G., Padilla, A., Skordis, C. :- Physics Reports 513 (2012)

[26] Chakraborty, S., Debnath, U., Ranjit, C. :- Eur. Phys. Jour. C 722101 (2012)

[27] Ranjit, C., Rudra, P., Kundu, S. :- Astrophys. Space Sci. 347423 (2013)

[28] Cohen, A., Kaplan, D., Nelson, A. :- Phys. Rev. Lett. 824971 (1999).

[29] Sahni, V., Shtanov, Y. :- JCAP 0311014 (2003).

[30] Cai, R. G. :- Phys. Lett. B 657228 (2007).

[31] Wei, H., Cai, R. G. :- Phys. Lett. B 660113 (2008).

[32] Choudhury, T. R., Padmanabhan, T. :- Astron. Astrophys. 429807 (2007). 
[33] Tonry, J. L. et al. :- ApJ 5941 (2003).

[34] Barris, B. J. et al. :- ApJ 602571 (2004).

[35] Lu, J. et al. :- Phys. Lett. B 66287 (2008).

[36] Dao-Jun, L., Xin-Zhou, L. :- Chin. Phys. Lett. 221600 (2005).

[37] Dvali, G. R., Gabadadze, G., Porrati, M. :- Phys. Lett. B 484112 (2000).

[38] De Felice, A., Tsujikawa, T. :- arXiv: 1002.4928 [gr-qc].

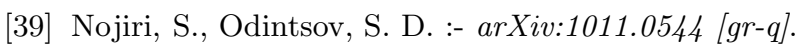

[40] Clifton, T., Barrow, J. :- Phys. Rev. D 72103005 (2005).

[41] Yerzhanov, K. K. et al. :- arXiv:1006.3879v1 [gr-qc] (2010).

[42] Nojiri, S., Odintsov, S. D. :- Phys. Lett. B 6311 (2005).

[43] Antoniadis, I., Rizos, J., Tamvakis, K. :- Nucl. Phys. B 415497 (1994).

[44] Horava, P. :- JHEP 0903020 (2009).

[45] Brans, C., Dicke, H. :- Phys. Rev. 124925 (1961).

[46] Rudra, P., Debnath, U., Biswas, R. :- Astrophys. Space Sci. 33953 (2012).

[47] Rudra, P. :- Astrophys. Space Sci. 342579 (2012).

[48] Chowdhury, R., Rudra, P. :- Int. J. Theor. Phys. 52489 (2013).

[49] Rudra, P., Ranjit, C., Kundu, S. :- Astrophys. Space Sci. 347433 (2013).

[50] Zlosnik, T.G., Ferreira, P.G., Starkman, G.D. :- Phys. Rev. D 75044017 (2007).

[51] Zlosnik, T.G., Ferreira, P.G., Starkman, G.D. :- Phys. Rev. D 77084010 (2008).

[52] Jacobson, T., Mattingly, D. :- Phys. Rev. D 64024028 (2001).

[53] Jacobson, T., Mattingly, D. :- Phys. Rev. D 70024003 (2004).

[54] Garfinkle, D., Jacobson, T. :- Phys. Rev. Lett. 107191102 (2011).

[55] Linder, E. V., Scherrer, R. J. :- Phys. Rev. D 80023008 (2009).

[56] Barrow, J. D. :- Phys. Rev. D 85047503 (2012).

[57] Zuntz, J., Zlosnik, T.G., Bourliot, F., Ferreira, P.G., Starkman, G.D. :- Phys. Rev. D 81 104015 (2010).

[58] Li, B., Fonseca Mota, D., Barrow, J.D. :- Phys. Rev. D 77024032 (2008).

[59] Gasperini, M. :- Class. Quant. Grav. 4485 (1987).

[60] Gasperini, M. :- Gen. Rel. Grav. 301703 (1998).

[61] Debnath, U. :- arXiv: 1310.2144v1 [gr-qc]

[62] Meng, X., Du, X. :- Phys. Lett. B 710493 (2012).

[63] Meng, X., Du, X. :- Comm. Theor. Phys. 57227 (2012).

[64] Sahni, V. et al. :- JETP 77 201(2003). 